\title{
Genome sequence and rapid evolution of the rice pathogen Xanthomonas oryzae pv. oryzae PXO99A
}

Steven L Salzberg*1, Daniel D Sommer ${ }^{1}$, Michael C Schatz ${ }^{1}$, Adam M Phillippy1, Pablo D Rabinowicz ${ }^{2,3}$, Seiji Tsuge ${ }^{4}$, Ayako Furutani4 ${ }^{4,5}$, Hirokazu Ochiai ${ }^{5}$, Arthur L Delcher ${ }^{1}$, David Kelley ${ }^{1}$, Ramana Madupu ${ }^{2,6}$, Daniela Puiu' ${ }^{1}$, Diana Radune ${ }^{2,6}$, Martin Shumway ${ }^{2,7}$, Cole Trapnell1, Gudlur Aparna 8 , Gopaljee Jha ${ }^{9}$, Alok Pandey ${ }^{8}$, Prabhu B Patil ${ }^{8}$, Hiromichi Ishihara10, Damien F Meyer ${ }^{11}$, Boris Szurek ${ }^{12}$, Valerie Verdier ${ }^{12}$, Ralf Koebnik12, J Maxwell Dow ${ }^{13}$, Robert P Ryan13, Hisae Hirata14, Shinji Tsuyumu13 ${ }^{13}$ Sang Won Lee ${ }^{15}$, Young-Su Seo ${ }^{15}$, Malinee Sriariyanum ${ }^{15}$, Pamela C Ronald ${ }^{15}$, Ramesh V Sonti ${ }^{8}$, Marie-Anne Van Sluys ${ }^{9,16}$, Jan E Leach ${ }^{9}$, Frank F White ${ }^{17}$ and Adam J Bogdanove ${ }^{11}$

Address: ${ }^{1}$ Center for Bioinformatics and Computational Biology, University of Maryland, College Park, MD 20742, USA, ${ }^{2}$ The Institute for Genomic Research, Rockville, MD 20850, USA, ${ }^{3}$ Institute for Genome Sciences, University of Maryland, Baltimore, MD 21201, USA, ${ }^{4}$ Laboratory of Plant Pathology, Kyoto Prefectural University, Sakyo, Kyoto 606-8522, Japan, ${ }^{5}$ Department of Genetic Resources, National Institute of Agrobiological Sciences, Kannondai, Tsukuba 305-8602, Japan, ${ }^{6}$ Current address: J. Craig Venter Institute, Rockville, MD 20850, USA, ${ }^{7}$ Current address: National Center for Biotechnology Information, National Institutes of Health, Bethesda, MD 20894, USA, ${ }^{8}$ Centre for Cellular and Molecular Biology, Council of Scientific and Industrial Research, Hyderabad, India, 9 Institute of Himalayan Bioresource Technology, Council of Scientific and Industrial Research, Palampur, India, ${ }^{10}$ Department of Bioagricultural Sciences and Pest Management, Colorado State University, Fort Collins, CO, USA, ${ }^{11}$ Department of Plant Pathology, Iowa State University, Ames, IA, USA, ${ }^{12}$ Institut de la Recherche pour le Developpement, 911 Av. Agropolis, Montpellier, 34090, France, ${ }^{13}$ BIOMERIT Research Centre, BioSciences Institute, University College Cork, Cork, Ireland, ${ }^{14}$ Graduate School of Natural Science \& Technology, Shizuoka University, 836 Ohya, Suruga-ku, Shizuoka, 422-8017, Japan, ${ }^{15}$ Department of Plant Pathology, UC Davis, Davis, CA 95616, USA, ${ }^{16}$ Departamento de Botânica, IB-USP, Sao Paulo, SP, Brazil and ${ }^{17}$ Department of Plant Pathology, Kansas State University, Manhattan, KS, USA

Email: Steven L Salzberg* - salzberg@umiacs.umd.edu; Daniel D Sommer - dsommer@umiacs.umd.edu;

Michael C Schatz - mschatz@umiacs.umd.edu; Adam M Phillippy - amp@umiacs.umd.edu;

Pablo D Rabinowicz - prabinowicz@som.umaryland.edu; Seiji Tsuge - s_tsuge@kpu.ac.jp; Ayako Furutani - a9920614@kpu.ac.jp; Hirokazu Ochiai - ochiaih@nias.affrc.go.jp; Arthur L Delcher - adelcher@umiacs.umd.edu; David Kelley - dakelley@umiacs.umd.edu; Ramana Madupu - rmadupu@jcvi.org; Daniela Puiu - dpuiu@umiacs.umd.edu; Diana Radune - dbushman@jcvi.org; Martin Shumway - shumwaym@ncbi.nlm.nih.gov; Cole Trapnell - cole@cs.umd.edu; Gudlur Aparna - aparna@ccmb.res.in; Gopaljee Jha - jmsgopal@yahoo.co.in; Alok Pandey - alok@ccmb.res.in; Prabhu B Patil - prabhubpatil@gmail.com; Hiromichi Ishihara - hiromichi.ishihara@colostate.edu; Damien F Meyer - dfmeyer@iastate.edu; Boris Szurek - boris.szurek@mpl.ird.fr; Valerie Verdier - valerie.verdier@mpl.ird.fr; Ralf Koebnik - koebnik@mpl.ird.fr; J Maxwell Dow - m.dow@ucc.ie; Robert P Ryan - r.ryan@ucc.ie; Hisae Hirata - hisaeh@agr.shizuoka.ac.jp; Shinji Tsuyumu - tsuyumu@agr.shizuoka.ac.jp; Sang Won Lee - drlee@ucdavis.edu; YoungSuSeo - yseo@ucdavis.edu; Malinee Sriariyanum - msriar@ucdavis.edu; Pamela C Ronald - pcronald@ucdavis.edu; Ramesh V Sonti - sonti@ccmb.res.in; Marie-Anne Van Sluys - mavsluys2004@yahoo.com; Jan E Leach - jan.leach@colostate.edu; Frank F White - fwhite@ksu.edu; Adam J Bogdanove - ajbog@iastate.edu

* Corresponding author

Published: II November 2008

BMC Genomics 2008, 9:534 doi:10.1186/147|-2164-9-534
Received: 29 October 2008

Accepted: II November 2008

This article is available from: http://www.biomedcentral.com//47I-2/64/9/534

(c) 2008 Salzberg et al; licensee BioMed Central Ltd.

This is an Open Access article distributed under the terms of the Creative Commons Attribution License (http://creativecommons.org/licenses/by/2.0), which permits unrestricted use, distribution, and reproduction in any medium, provided the original work is properly cited. 


\section{Correction}

Following the publication of the article 'Genome sequence and rapid evolution of the rice pathogen Xanthomonas oryzae pv. oryzae PXO99A. BMC Genomics 2008, 9:204' [1], the submitting author became aware that two co-authors had been omitted. Therefore, this article, which states the contributions that these authors made to the original article, has been submitted as a correction. We apologize for any inconvenience this oversight may have caused.

\section{Corrected author list}

Steven L Salzberg, Daniel D Sommer, Michael C Schatz, Adam M Phillippy, Pablo D Rabinowicz, Seiji Tsuge, Ayako Furutani, Hirokazu Ochiai, Arthur L Delcher, David Kelley, Ramana Madupu, Daniela Puiu, Diana Radune, Martin Shumway, Cole Trapnell, Gudlur Aparna, Gopaljee Jha, Alok Pandey, Prabhu B Patil, Hiromichi Ishihara, Damien F Meyer, Boris Szurek, Valerie Verdier, Ralf Koebnik, J Maxwell Dow, Robert P Ryan, Hisae Hirata, Shinji Tsuyumu, Sang Won Lee, Young-Su Seo, Malinee Sriariyanum, Pamela C Ronald, Ramesh V Sonti, Marie-Anne Van Sluys, Jan E Leach, Frank F White and Adam J Bogdanove.

\section{Corrected authors' contributions}

SLS, JEL, FFW, and AJB conceived the project. SLS, PDR, and AJB coordinated and oversaw the project. SLS and PDR managed all genomic sequencing. DP and MCS did the initial assembly of the genome. DR directed the sequence finishing and gap closure activities. MCS, AMP, and ALD created the final assembly. RM was in charge of the initial, semi-automated genome annotation. MCS, CT, and SLS carried out the overall structural analysis of the genome. PBP and RVS performed the whole genome alignments for phylogenetic analysis. DK, CT, DDS, and SLS compared the gene content of PXO99A and MAFF. CT and MVS analyzed IS elements. GA and RVS analyzed the adhesin locus. MCS, ALD, and SLS discovered and characterized the $212 \mathrm{~kb}$ duplication. FFW carried out the TAL effector analysis, assisted by RK and AJB. CT documented rearrangements in the $\mathrm{PXO} 99^{\mathrm{A}}$ genome relative to MAFF. DDS, SLS and RK investigated the CRISPRs. SeT, AF, and HO validated the MAFF assembly. SLS identified regions of possible lateral gene transfer. DK optimized annotation of hypothetical protein genes. SeT, AF, GA, GJ, AP, PBP, RVS, HI, DFM, BS, VV, JMD, RPR, HH, ShT, SWL, YS, MS, PCR, RVS, MVS, JEL, FFW, and AJB contributed to the manual annotation. SLS and AJB drafted the manuscript, assisted by PDR, SeT, GA, PBP, RVS, RK, MVS, JEL, and FFW. All authors approved the final manuscript.

\section{References}

I. Salzberg SL, Sommer DD, Schatz MC, Phillippy AM, Rabinowicz PD, Tsuge S, Furutani A, Ochiai H, Delcher AL, Kelley D, et al.: Genome sequence and rapid evolution of the rice pathogen Xanthomonas oryzae pv. oryzae PXO99A. BMC Genomics 2008, 9:204.

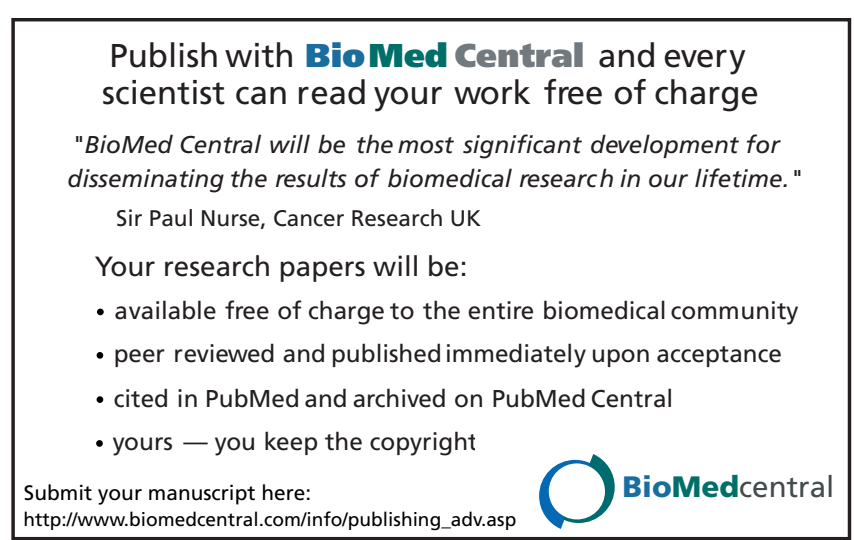

